ARTÍCULO

\title{
Modelos de competencias sobre formación política: aporte a la formación inicial docente en educación ciudadana
}

\section{RESUMEN}

Este artículo tiene por objetivo analizar los procesos de construcción y cristalización teórica con relación a las competencias sobre formación política en el debate de la didáctica política germana. Para alcanzar este objetivo se examinaron tres modelos de formación política: el modelo presentado por la Sociedad para la Didáctica y Formación Política Juvenil y Adulta de Alemania (Modelo GPJE); el modelo de competencia política propuesto por el equipo de Georg Weißeno; y el modelo austriaco de competencias de formación política. Sobre esta base, se extraen implicaciones para pensar la formación inicial docente en la educación de la ciudadanía para Chile. Entre los principales resultados se encuentran: La formación inicial de profesores requiere a nivel teórico y metodológico articular de manera dialéctica: el ámbito de saber de la especialidad como componente nuclear y el ámbito de competencia didáctica en la formación ciudadana.

PALABRAS CLAVE

formación política; educación ciudadana; modelo de competencias; formación inicial docente.

Universidad Católica del Maule, Maule, Chile. 


\title{
COMPETENCY-BASED MODELS REGARDING POLITICAL EDUCATION: A CONTRIBUTION TO INITIAL TEACHER FORMATION IN CITIZEN EDUCATION
}

\begin{abstract}
This article aims to analyze the processes of construction and theoretical solidification of competencies related to political education in the debate on German political didactics. To achieve this task, three models of political education were examined: the model advanced by the German Society for Child and Adult Didactics and Political Education (GPJE Model); the model of political competency advanced by Georg Weißeno's team; and the Austrian model of political education competencies. A number of implications are drawn from this discussion regarding initial teaching training in the Chilean civic education. The main results include: On a theoretical and methodological level, teachers' initial training has to articulate the domain of the specialist knowledge as core component and the domain of didactic competency in citizenship education.
\end{abstract}

KEYWORDS

political education; citizen education; competency-based model; initial teacher formation.

\section{MODELOS DE COMPETÊNCIA NA EDUCAÇÃO POLITICA: CONTRIBUIÇÕES PARA A FORMAÇÃO DOCENTE INICIAL EM EDUCAÇÃO CIDADÃ}

RESUMO

Este artigo pretendeu analisar os processos de construção teórica em relação às competências sobre educação política no debate sobre a didática política alemã. Para isso, três modelos de educação política serão analisados: o modelo apresentado pela Sociedade de Didática e Educação Política para Jovens e Adultos da Alemanha (Modelo GPJE); o modelo de competências políticas proposto pela equipe de Georg Weißeno; e o modelo austríaco de competências de educação política. A partir destes modelos, há implicações para se pensar a formação docente inicial em educação cidadã para o Chile. Entre os principais resultados está a constatação de que a formação docente inicial exige, em nível teórico e metodológico, uma articulação dialética entre o campo do conhecimento da especialidade como componente curricular e o campo da competência didática na educação cidadã.

PALAVRAS-CHAVE

educação política; educação cidadã; modelo de competências; formação docente inicial. 


\section{INTRODUCCIÓN}

Con la publicación de los estándares sobre formación política en Alemania (2004) se inicia una discusión académica en la didáctica política en países de lengua germana. La formación política se entiende como la promoción de principios de la democracia, la tolerancia y la capacidad crítica, que en palabras de Thomas Hellmuth y Cornelia Klepp (2010) buscan "promover ciudadanos activos, que se introducen en procesos de socialización en verdad conscientes y que a través del juicio político y la capacidad crítica pueden contribuir al desarrollo posterior de una sociedad democrática” (Hellmuth y Klepp, 2010, p. 13).

A diferencia de la categoría "educación ciudadana" que adquiere popularidad en países anglófonos, así como en la Comunidad Europea y países de América Latina a partir de la década de los noventa del siglo XX, la formación política en Alemania es el resultado de una profunda discusión sobre el rol y finalidad que juega la educación política dentro y fuera del sistema escolar después de la segunda guerra mundial. En efecto, desde los años 60 del siglo pasado comienzan gradualmente las universidades alemanas a incorporar cursos sobre formación política, promoviendo una discusión y producción teórica sobre esta temática en la formación de profesores (Detjen, 2007; Hellmuth y Klepp, 2010; Massing, 2007; Sander, 2007).

El presente artículo delimita su estudio a los modelos de competencias en formación política. A través de la revisión de la literatura hemos encontrado una intensa discusión teórica que se registra a partir del año 2004 y que despliega en una serie de publicaciones tales como: "Politikkompetenz. Was Unterricht zu leisten hat" ["Competencia política. Qué tiene que hacer la enseñanza”], publicado el 2008 por Georg Weißeno. En ella esboza las tareas que se desprenden a nivel teórico y de la praxis para fundamentar la noción de competencia política, dos años después aparece el libro "Konzepte der Politik - Ein Kompetenzmodell" ["Conceptos de política - Un modelo de competencia"], allí se hace un exposición fundamentada sobre las bases de la competencia política como el saber político escolar en el sentido de los estándares. Ese mismo año la revista Politik und Wirtschaft Unterrichten de la editorial Wochenschau publica un número especial con el título "Kompetenzen im Unterricht" ["Competencias en la clase"]. El 2011 publica Thomas Goll el libro "olitische Basis- und Fachkonzepte" ["Conceptos base y disciplinares en Política"] y el 2012 aparece "Politische Handeln: Modelle, Möglichkeiten, Kompetenen" ["Acción política: Modelos, posibilidades y competencias"].

Todos estos libros buscan fundamentar a nivel epistemológico, de la psicología del aprendizaje y a nivel curricular las competencias de formación política.

El objetivo del presente estudio es analizar qué tipo de conocimiento se ha producido al interior del debate sobre los modelos en formación política y qué implicancias se pueden extraer para pensar la formación inicial de profesores en la especialidad de formación ciudadana en Chile.

En el análisis de la fundamentación de estos modelos hemos encontrado un hilo conductor, que nos permite sostener que estamos frente a un proceso de construcción y cristalización teórica en torno a las competencias de formación política. La intención es visibilizar los presupuestos teóricos que sustentan a estos modelos, 
con ello buscamos comprender ¿qué competencias de formación política proponen en Alemania y Austria para la formación política?, ¿cuáles son los supuestos teóricos en los cuales se sustentan los modelos de competencias sobre formación política?, ¿estos modelos se contraponen o complementan?, ¿cuáles son sus diferencias y puntos de encuentro?, así como saber ¿cuáles son sus nudos críticos? Ahora bien, la relevancia del estudio es recuperar esta teorización elaborada en la discusión de la didáctica política y conectarla con la formación inicial de profesores para la formación ciudadana en Chile.

Aquí se hace necesario indicar que en Chile y, en general, en América Latina la temática de la formación inicial docente en educación ciudadana ha sido descuidada. El estudio de la UNESCO señala que, si bien el curso de educación ciudadana está presente en el currículum de Educación básica y en la especialidad de Historia y Ciencias Sociales en la educación media de los países latinoamericanos. Sin embargo, la presencia de la educación ciudadana en el currículum de la formación de docentes de los países en estudio es tenue y aún muy variable (UNESCO, 2017, p. 39). A nivel curricular se observa que la educación ciudadana ocupa un lugar marginal en la formación inicial docente. "Esto se evidencia en la baja cantidad de cursos que se hacen cargo de ella, el escaso tiempo con que se cuenta para desarrollarla y la insuficiente relación con la práctica" (UNESCO, 2017, p. 92).

Los estudios han identificado que uno de los grandes problemas de la educación ciudadana consiste en que los docentes no están suficientemente preparados para desarrollar esta tarea, ni han logrado incorporar el enfoque pedagógico que la educación ciudadana para la democracia requiere, transformándose así en uno de sus eslabones más débiles. (UNESCO, 2017, p. 16)

De ello se desprende que en América Latina nos enfrentamos no solo a un problema práctico de cómo enseñar esta asignatura sino, ante todo, nos muestra la existencia de un vacío teórico y la necesidad de abrir espacios de reflexión pedagógica en el ámbito de la educación ciudadana de la formación inicial docente.

E1 hilo conductor del presente artículo se estructura de la siguiente manera. En un primer momento se hace una presentación de los puntos nucleares que caracterizan a cada uno de los tres modelos. Con ello, se busca comprender básicamente qué propone cada modelo, cuáles son sus supuestos y rasgos distintivos.

En un segundo momento se realiza una comparación de los modelos. Aquí hemos encontrado elementos que nos permiten sostener que estamos frente a un proceso de construcción y cristalización teórica en torno a las competencias de formación política. Es decir, entre los modelos estudiados se identifican procesos de profundización, contrastación y diferenciación, tanto a nivel normativo como procedimental.

En un tercer momento, se extraen de los modelos de competencias de formación política dos contribuciones para la discusión teórica de la formación inicial docente en formación ciudadana en Chile:

- El ámbito del saber, concebido como un componente nuclear para la formación inicial docente en formación ciudadana;

- El ámbito de competencia didáctica en formación ciudadana. 


\section{MODELO DE ÁMBITOS DE COMPETENCIAS DE LA FORMACIÓN POLÍTICA DE LA GPJE (2004)}

El primer modelo de competencias sobre formación política fue elaborado por la Sociedad para la Didáctica y Formación Política Juvenil y Adulta, denominada en alemán Gesellschaft für Politikdidaktik und politische Jugend-und Erwachsenenbildung (GPJE), publicado el año 2004 con el título Anforderungen an Nationale Bildungsstandards für den Fachunterricht in der Politischen Bildung an Schulen. Ein Entwurf. El equipo de investigadores que participó en su elaboración son Joachim Detjen, Hans-Werner Kuhn, Dagmar Richter, Wolfgang Sander y Georg Weißeno. El documento es una contribución al desarrollo de estándares en educación para la asignatura de formación política en la escuela de Alemania.

La propuesta de GPJE contiene dos planteamientos centrales que ponen el acento en el nivel normativo de la propuesta de formación política y que abrirá un discusión académica en la didáctica política.

El primer planteamiento tiene que ver con la meta y su perspectiva. La propuesta de la formación política deja claramente establecido que no es una asignatura que asocie sin más elementos de educación y elementos de política, por el contrario, se subraya desde el principio que la meta de la formación política es contribuir al desarrollo de la "politische Mündigkeit"(GPJE, 2004,p. 9). En esta línea, la propuesta de formación política se conecta a nivel normativo con la tradición alemana contenida en los fines de la "formación"("Bildung"), que está dirigida al desarrollo de las capacidades de autodeterminación, organización y solidaridad (Klafki, 1991; Sander, 2015).

La "politische Mündigkeit" se traduce como "autonomía política", ella es descrita en el documento de GPJE en los siguientes términos: "La autonomía política es una imprescindible meta de la formación escolar, desde una perspectiva personal es una condición para la participación exitosa, pero ella es también, desde la perspectiva social común para el mantenimiento y desarrollo continuo de una cultura política democrática y de un sistema político democrático" (GPJE, 2004, p. 9). La autonomía política está asociada al desarrollo de capacidades analíticas, en esta línea se subraya que a través de la formación política "la escuela promueve la capacidad de los jóvenes para orientar adecuadamente en la economía moderna y en la sociedad, para juzgar competentemente preguntas políticas y problemas sobre bases democráticas y para comprometerse en asuntos públicos"(GPJE, 2004, p. 9). En este marco, la formación política se dirige a promover capacidades democráticas.

El segundo planteamiento está en relación con el modelo de competencias propuesto por la GPJE. La definición de competencia aceptada dentro la discusión teórica sobre el modelo por competencias en formación política es la elaborada por Franz E. Weinert, quien acuña este concepto desde la psicología del aprendizaje, para él

$\mathrm{El}$ término de competencia se refiere a capacidades cognitivas que poseen los individuos o que a través de ellas pueden aprender capacidades cognitivas para resolver determinados problemas, así como con estas disposiciones y habilidades motivacionales, volitivas y sociales articuladas pueden utilizar para solucionar problemas en situaciones variables con éxito y responsabilidad. (Hellmuth y Klepp, 2010, p. 99-100) 
El modelo de competencias de la GPJE se organiza en tres ámbitos de competencias, la capacidad del juicio político, la capacidad de la acción política y las capacidades metodológicas. A lado de estos tres ámbitos de competencias se ubica un cuarto ámbito que en sentido estricto no es una competencia, sino un saber, denominado como saber de interpretación conceptual (Hedinger, 2018, p. 59-60).

Un aspecto central del modelo GPJE tiene que ver con el lugar y función que cumple el ámbito del saber, este se presenta como condición y mediación para el desarrollo de las competencias. El saber funciona como un modelo de explicación e interpretación, compuesto por conceptos fundamentales.

Ahora bien, ¿Cuál es ese saber? La asignatura de formación política se apoya en una definición amplia de política, caracterizada por su sentido social, que está en relación con aquellas "preguntas básicas y problemas de la convivencia social general". A ella pertenecen la política, la economía, la convivencia social y el derecho (Cf. GPJE, 2004).

La formación política utiliza la política como un concepto útil de trabajo, es comprendida a través de dimensiones. "La dimensión formal de la política (Polity)", hace referencia a la estructura del orden político, compuesto por la constitución política del Estado, las instituciones políticas, el funcionamiento del sistema político. "La dimensión del contenido de la política (policy)", señala las metas y tareas de la política como de la formación de las relaciones sociales así como la competencia de intereses políticos e ideológicos, aquí se describe las características que poseen los actores, sus valores, opciones ideológicas y sus metas. "La dimensión de proceso de la política (politics)", describe las acciones de los actores políticos y sus procesos formales e informales, se analiza los conflictos políticos y la formación de consensos. Tiene que ver con el análisis de la coyuntura política.

Dentro de la psicología cognitiva estos conceptos se entienden como esquemas, modelos mentales a partir de los cuales los estudiantes comprenden y explican la sociedad en la que viven. El saber no se entiende como adquisición de conocimientos, sino mucho más, es un saber de interpretación que permite a los jóvenes comprender la lógica interna de las instituciones, los modelos de organización social y política de la democracia como sus principios (Cf. GPJE, 2004, p. 14). A partir de este trasfondo se puede caracterizar los tres ámbitos de competencias.

La capacidad del juicio político es el primer ámbito de competencia, ella ocupa un lugar dominante, pues permite desarrollar la capacidad cognitiva del estudiante. A través de la comprensión y estructuración de los juicios analíticos y juicios de valor, los estudiantes pueden diferencian, contrastan y analizan sus planteamientos, argumentos y juicios de valor del estudio de determinados temas y problemas. En cambio, la capacidad de la acción política se relaciona con las competencias comunicativas, con ellas pueden los estudiantes: Representar convincentemente sus propias opiniones políticas así como de la posición de la minoría; estar capacitado para entrar en controversias políticas conflictivas y poder llegar a compromisos, expresar su opinión política a través de formulación y argumentación de preguntas políticas, económicas y sociales a través de medios, tales como cartas, páginas en Internet y otros medios (Cf. GPJE, 2004, p. 17). 
Por su parte, la capacidad metodológica de la formación política hace referencia a las competencias de lectura, planificación y organización personal, se mencionan técnicas de trabajo (interpretación de cuadros y caricaturas, técnicas de presentación de pesquisas o sondeos, así como la interpretación de textos, la producción de medios y la planificación y realización de proyectos), que se promueve también en otras asignaturas (Cf. GPJE, 2004, p. 18).

Así el modelo GPJE, caracterizado por sus tres ámbitos de competencias (capacidad del juicio político, capacidad de la acción política y capacidades metódicas) y el saber de interpretación conceptual, es una propuesta que se construyó a partir del resultado del análisis de las competencias y de la sistematización del desarrollo de los planes de estudio y de la praxis escolar (Redwanz, 2013, p. 147-148), que coloca como núcleo central la capacidad del juicio político, pues el saber de interpretación conceptual como la capacidad de la acción política y las capacidades metodológicas se integran en ella. Con ello se puede afirmar que la concreción de la "politische Mündigkeit" en capacidades analítica pasa necesariamente por la promoción de la capacidad del juicio político (Ziegler, 2018, p. 104).

\section{EL MODELO DE COMPETENCIA POLÍTICA}

El modelo de competencia política fue publicado el año 2010 por Georg Weißeno, Joachim Detjen, Ingo Juchler, Peter Massing y Dagmar Richter con el título Konzepte der Politik - Ein Kompetenzmodell. El equipo se propuso elaborar una fundamentación normativa de la competencia política para responder a aspectos centrales que quedaron implícitos en el modelo GPJE, específicamente con relación a la noción saber de interpretación conceptual, descrito como un saber que se refiere a conceptos básicos para el entendimiento de la política, economía, sociedad y derecho (GPJE, 2004, p. 17), el significado y fundamentación de este concepto queda, sin embargo, abierto y frente a este déficit en relación al saber disciplinar el equipo dirigido por George Weißeno se propuso fundamentar un modelo de competencia política a nivel teórico y sistemático.

En el presente apartado subrayamos dos aspectos, por una parte se busca caracterizar la estructura argumentativa del modelo y, por otra, se presenta los componentes del mismo.

Los autores comprenden la competencia en la línea de Weinert, es decir como competencia psicológica, que apunta a promover capacidades y habilidades cognitivas. A partir de este marco, el equipo de Weißeno delimita su campo de acción dentro de la denominada "Fachkompetenz" (competencia disciplinar) y sobre ella formula su modelo. Es decir, su propuesta se concentra en una competencia política relacionada con el contenido, Dagma Richter (2010, p. 59) precisa la denominación dentro del enfoque por competencias, se elabora un modelo para una dimensión de la competencia, que pertenece al saber disciplinar o si se prefiere un modelo de competencia para el ámbito del saber.

La fijación concreta en este tipo de competencia tiene una gran relevancia para el contenido de los estándares y los contenidos de aprendizaje que los estu- 
diantes deben poseer, así como permite a nivel teórico examinar por qué ese saber se tiene que promover.

Se presenta un modelo teórico de conceptos base y disciplinares, en el que la dimensión disciplinar se pueda concretar. "El modelo se apoya en el enfoque psicológico cognitivo del aprendizaje de contenidos y conectan con la concretización de contenidos a través de una selección didáctica fundamentada en el saber de la didáctica política" (Weißeno, 2012, p. 166).

Los conceptos base hacen referencia al contenido de las disciplinas y constituyen la dimensión de la competencia relacionada con el saber de la especialidad, busca ser comprendida como un saber conceptual que le brindará al estudiante la oportunidad de ampliar su capacidad de comprensión, por medio de categorías, teorías, modelos de interpretación, entre otros.

"Los conceptos base son por definición conceptos disciplinares que en su aplicación poseen, ante todo, funciones didáctico específicas" (Massing, 2008, p. 191).

Los conceptos base hacen alusión a un "saber conceptual", que explica cómo los estudiantes a partir de los términos y conceptos de un campo científico pueden construir relaciones semánticas para describir y explicar fenómenos y elaborar nuevas ideas a partir de la asignatura (Richter, 2010).

Detjen hace una interesante aclaración cuando se refiere a los conceptos base, estos se ubican lógicamente en un nivel de abstracción de una disciplina o dominio y se diferencian o distinguen de otros conceptos base, así como tienen la capacidad de intersección (Cf. Detjen, 2011, p. 45-48). En cambio, los conceptos disciplinares se ubican en un nivel más concreto y se ordenan al interior de un concepto base. Los conceptos disciplinares caracterizan un ámbito nuclear del saber de dominio en interés didáctico. Ellas toman la organización y las funciones de orden para los contenidos de los temas de clase, así como para el aprendizaje. En general se origina entonces un entramado de conceptos, una red estructurada, que describen fenómenos políticos, procesos, acontecimientos para analizar y reflexionar.

Weißeno se apoya en la comprensión de "Civic Literacy" propuesta por R. Bybee para orientar el modelo de conceptos disciplinares en el dominio de la política de la educación básica, descritos en cuatro niveles:

- Alfabetización cívica nominal, que hace referencia a conocimientos y temas políticos, así como nombres y palabras mal comprendidas por los estudiantes (Ej. Angela Merkel es reina de Alemania);

- Alfabetización cívica funcional, denota el uso correcto de los términos (Ej. Elecciones, que hace referencia a un saber fáctico);

- Alfabetización cívica procedimental y conceptual, se refiere a la comprensión de conceptos centrales políticos y el significado de los procesos políticos, presentación de relaciones entre hechos, conceptos y principios (Ej. Parlamento con voto de censura constructivo);

- Alfabetización cívica multidimensional, comprensión de peculiaridades del pensamiento político y capacidad para ordenar de manera analítica aspectos económicos, sociales, y culturales para explicar con coherencia fenómenos y problemas en la sociedad que vive (La lógica propia del concepto poder en la economía y la política) (Cf. Weißeno, 2008, p. 14). 
El enfoque de la Civic Literacy y los niveles explican que la competencia política no puede derivarse exclusiva y únicamente de la ciencia política, por el contrario, "el saber disciplinar adquirido en la educación escolar se constituye recién a través de la construcción didáctica del saber de las ciencias políticas" (Weißeno et al., 2010, p. 19). Este proceso de construcción tiene que legitimar la selección, la compresión y secuenciación del saber. En este punto, Dagma Richter sostiene que la orientación de la competencia al ámbito del saber se relaciona con la funcionalidad, es decir, si los estudiantes pueden utilizar su saber flexible para analizar un problema en una determinada situación y para ello el enfoque Civic Literacy y los niveles le ofrecen al profesor criterios para valorar si la salida de un tema es importante o no.

Después de haber caracterizado la estructura argumentativa del modelo, pasamos a presentar sus componentes básicos.

El concepto de política que sustenta el modelo de competencia política se describe como "una acción que se produce en un marco de formas determinadas de regulaciones comunes vinculantes en y entre grupos de personas hacia el bienestar de las personas" (Detjen, 2011, p. 46). A partir de este concepto Weißno, Detjen, Massing y Richter extraen tres conceptos base que tienen el mismo rango y que constituyen la misma política, ellas son: "orden", "decisión" y "bien común". Los conceptos base funcionan como principios o ideas rectoras de la asignatura y son consideradas imprescindibles para la comprensión política.

"Orden" es un concepto que denota un nivel de abstracción general, porque tiene que ver con una condición para toda convivencia humana. En sentido estricto "el orden político" presenta el marco, dentro el cual la acción política y los procesos de decisión pueden desenvolverse. La función más importante del orden político estriba en que el orden logra "fiabilidad", hace que los procesos políticos sean por lo menos calculables y previsibles y coloca arbitramiento a las barreras. Por ello contiene un elemento normativo. El término "Orden político" se equipara a otras categorías como: Sistema de gobierno o sistema político.

$\mathrm{Al}$ interior de este concepto base se organizan diez conceptos disciplinares, que se encuentran descritos en el Cuadro 1, desde el concepto disciplinar de "democracia" a "Estado".

"Decisión" es un segundo concepto base de la política y contiene un acento normativo, porque es una consecuencia de las reflexiones y los argumentos para fundamentar una toma de decisión. Aquí se sostiene que la capacidad de decisión es importante en una sociedad democrática, que requiere de las capacidades analíticas para comprender y analizar los procesos. Es fundamental precisar los principios y criterios para la toma de decisiones tanto a nivel descriptivo como normativo.

"El bien común" es el tercer concepto base, se caracteriza por su carácter normativo y de abstracción, debido que caracteriza la meta general o las metas y valores comunes deseados dentro de una comunidad política. Apunta a los valores, bienes y condiciones para alcanzar el bienestar humano dentro la sociedad democrática, por ello apunta al sentido y meta de la política.

Así descrito, el modelo de competencia política propuesto por el equipo de Weißeno muestra la importancia de los conceptos base y disciplinares en el ámbito 


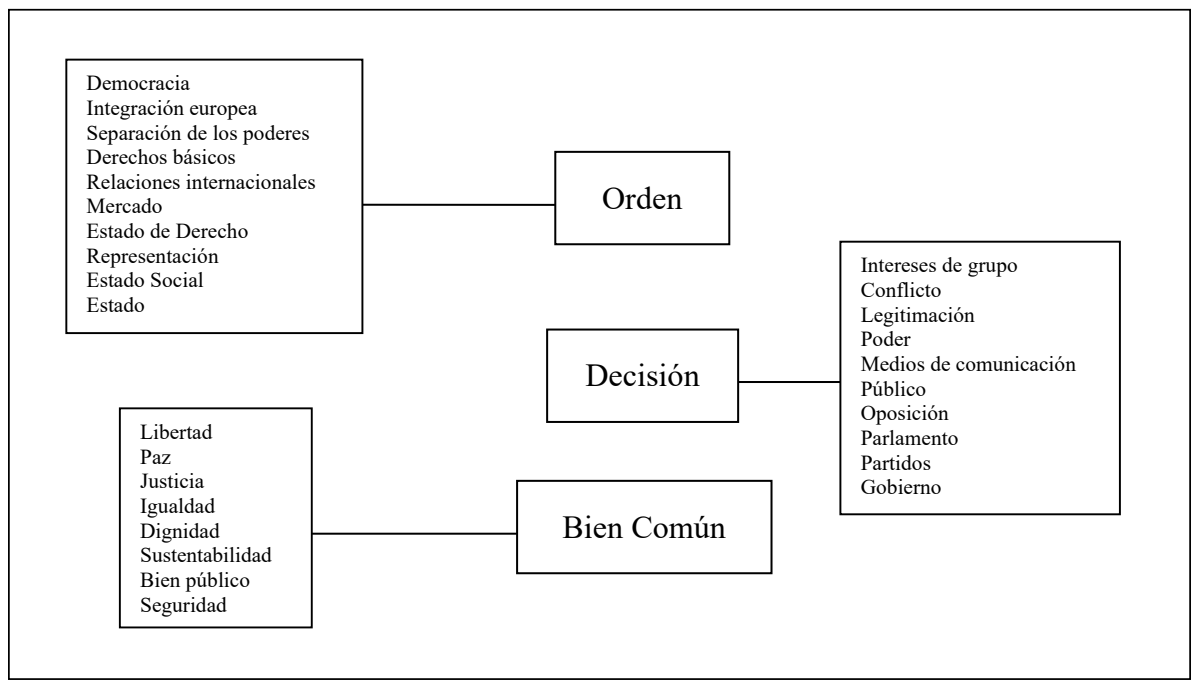

Cuadro 1 - Conceptos base y disciplinares del modelo de la competencia política.

Fuente: Richter, 2010.

del saber de la competencia política, visibiliza la complejidad de ese proceso, pues no se reduce a formas de aprendizaje, sino también a la definición normativa de los contenidos, es decir, ¿qué saber se debe enseñar primero y cuál después?, ¿¿cuáles son los criterios a partir del cual se toman esas decisiones para organizar los procesos de enseñanza y aprendizaje?

La discusión sobre los conceptos base ha permitido fundamentar la formación política y la didáctica política como un ámbito interdisciplinar que no se reduce a la política, ni a las ciencias sociales, ni a la filosofía, ella posee su propio estatuto epistemológico (Lobatón, 2016; Massing 2011).

El grupo de Weißeno sustenta a partir de su concepto de política tres conceptos base, los cuales son orden, decisión y bien común. El aporte de este modelo se encuentra en su estructura y funcionalidad, que permite a los estudiantes construir los conceptos base y disciplinares a partir de la comparación de las estructuras del saber con la comprensión y valoración de una situación concreta política. Es decir, a partir de la correspondencia entre los contenidos de estructura de enseñanza (relacionado con los conceptos disciplinares) y las estructuras cognitivas que posee el estudiante puede comprender y explicar fenómenos y preguntas del dominio (Richter, 2010, p. 61-62). Para alcanzar ese cometido, los pasos que se siguen son los siguientes: Se explica la esencia del concepto, se profundiza ese concepto desde las ciencias políticas, se identifican los errores del concepto, posteriormente se hace una conexión con los otros conceptos disciplinares y se ejemplifican con temas relacionados a su ciclo (Weißeno et al., 2010). Ello estructura una red conceptual que permite a los estudiantes comprender y analizar los temas y fenómenos políticos estudiados. 


\section{EL MODELO DE COMPETENCIAS ESTRUCTURAL EN FORMACIÓN POLÍTICA}

El año 2008 financia el Estado austriaco el proyecto denominado "modelo de competencias estructural en formación política”, que congregó varios especialistas de didáctica política como funcionarios del ministerio de educación. Resultado de ese proyecto se publicó el libro "Die durch politische Bildung zu erwerbenden Kompetenzen. Ein Kompetenz-Strukturmodell” (2008), elaborado por Reinhard Krammer, Cristoph Kühberger y Elfride Windischbauer. Allí se establece que la meta de la formación política es "promover ciudadanos austriacos autónomos, con capacidad de elegir desde bases sociales y político-democráticas y ello tendría que adquirirse durante su socialización escolar" ( $C f$. Krammer, 2013, p. 15). Al fundamentar este modelo, Krammer (2013) reconoce que un modelo de competencias es un ideal de la organización de la escuela. Sin embargo, es consciente de su contribución en la situación actual de la práctica educativa, pues ofrece elementos teóricos, didácticos y organizativos para orientar el proceso de enseñanza y aprendizaje. Este planteamiento se encuentra tanto en el modelo de la GPJE como en el modelo en la competencia de la formación política.

La competencia se entiende al interior de este modelo como capacidades, habilidades y disposiciones internas que permiten resolver determinados problemas. En esta línea, se apoyan en el concepto de competencia de Weinert. Se subraya que las competencias de la formación política en las clases tienen que ver con la capacidad autorreflexiva, se sostiene que "reflexionar es pensar políticamente"(Krammer,2013,p. 16) y para promover el pensamiento y acción reflexiva se requiere elementos teóricos y metodológicos.

El modelo de competencias estructural de formación política está organizado en cuadro ámbitos, competencia de la estructuración del saber (Sachkompetenz), competencia del juicio político (Urteilskompetenz), competencia metódica (Methodekompetenz) y competencia de la acción política (Handlungskompetenz).

El planteamiento central del modelo de competencias estructural es que sostiene que los procesos de aprendizaje no siempre se promueven en una sola área de competencias, sino, por el contrario, se desarrollan en la interacción simultánea de los diferentes ámbitos de competencias.

¿Qué papel juega dentro este modelo de competencias el saber? Los autores sostienen que si se aborda un tema, fenómeno o problema político, los estudiantes para realizar su análisis político requerirán manejar conocimientos, conceptos, teorías y modelos que permita su análisis, de esta manera, el saber no tiene un valor en sí mismo, como adquisición de conocimientos en términos descriptivos, sino se entiende el saber como un "saber de trabajo", es decir, tiene un carácter instrumental, permite realizar procesos de aprendizaje político necesario y con ello se avanza en el desarrollo de competencias políticas.

La competencia del juicio político (Politische Urteilskompetenz) busca promover la capacidad, habilidad y disposición hacia un juicio independiente, justificado, a nivel objetivo o de valor para la toma de decisiones en relación a problemas y controversias. En la Tabla 1 se muestra cómo el modelo austriaco operacionaliza la concreción de esta competencia. 
Tabla 1 - Parte de las competencias para examinar las decisiones y juicios políticos.

\begin{tabular}{l|ll}
\hline \multicolumn{2}{c}{ Se tiene que comprender, examinar y } \\
\multicolumn{2}{c}{ valorar las siguientes decisiones y juicios políticos. } \\
\hline $\begin{array}{l}\text { Ámbito de la cualidad } \\
\text { de prueba, de la } \\
\text { categorización y } \\
\text { clasificación }\end{array}$ & - & $\begin{array}{l}\text { Investigar la fundamentación del siguiente juicio } \\
\text { Distinguir un juicio previo, de un prejuicio y de un juicio fundamentado } \\
\text { Examinar el siguiente juicio sobre su compatibilidad con los } \\
\text { Derechos Humanos. }\end{array}$ \\
\hline $\begin{array}{l}\text { Ámbito de la declaración } \\
\text { de intereses y el estado de } \\
\text { situación }\end{array}$ & - $\begin{array}{l}\text { Comprender los puntos centrales y perspectivas de los actores que } \\
\text { participan en los problemas políticos, controversias y conflictos } \\
\text { Ordenar las posiciones políticas controversias, juicios y decisiones de } \\
\text { personas, grupos o partidos identificando sus valores políticos y sus } \\
\text { bases filosóficas y luego valorar sus decisiones } \\
\text { Interrogar posiciones controvertidas con la toma de decisiones } \\
\text { políticas por dependencia de intereses económicos, políticos, } \\
\text { culturales y religiosos }\end{array}$ \\
\hline $\begin{array}{l}\text { Ámbito del dictamen del } \\
\text { juicio, sus consecuencias y } \\
\text { efectos de la decisión política }\end{array}$ & $\begin{array}{l}\text { Conocer que a través de juicios políticos/decisiones se ha } \\
\text { considerado y descuidado intereses, evaluar las consecuencias de las } \\
\text { consecuencias y de los juicios/decisiones referidos }\end{array}$ \\
\hline
\end{tabular}

Fuente: Krammer, 2013, p. 19.

El modelo austriaco establece tres ámbitos para examinar las decisiones y juicios políticos. El ámbito de la cualidad de la prueba en el que se sustenta el juicio, precisando si estamos frente a un juicio o no y si el juicio presentado está fundamentado. El ámbito de las declaraciones de intereses, ella busca conocer y analizar los puntos centrales y perspectivas de los actores involucrados. Es decir, identifica los intereses de los actores, sus posiciones y valores, ahí se busca comprender en qué consiste la controversia, los factores e intereses que interactúan como los argumentos que se sostienen al respecto. Finalmente, el ámbito del dictamen hace referencia a la evaluación de los juicios políticos tomando en consideración las consecuencias directas e indirectas que se desprenden del mismo.

La competencia de la acción política (Politische Handlungskompetenz) es entendida como

la capacidad, habilidad y la disposición para formular y articular sus propias posiciones, entender las posiciones políticas de otros, buscar soluciones a los problemas estudiados. La competencia de la acción política apunta a promover la disposición al compromiso, la capacidad de comunicación, tolerancia y llegar a acuerdos. (Kühberger, 2010, p. 48)

Las competencias metodológicas (Politische Methodenkompetnez) comprenden habilidades para usar métodos orales, escritos, visuales y otros medios modernos con el fin de articular su reflexión sobre asuntos políticos, sociales y legales relevantes para la sociedad, así como crear manifestaciones propias para adquisición y valoración de información (Cf. Kühberger, 2010, p. 48). 
La competencia de la estructuración del saber político (Politische Sachkompetenz) se refiere a las capacidades, habilidades y disposiciones que son necesarios para comprender términos, categorías o conceptos de la política y sobre ella poder desarrollar una actitud crítica del tema estudiado.

El modelo estructurado de competencias conceptualiza los conceptos bases como ideas orientadoras o ideas básicas. Con la ayuda de ellas pueden los estudiantes ordenar y estructurar el saber político. A diferencia del modelo de competencia política, el modelo austriaco subraya tres dimensiones de la política como los conceptos base de la formación política, a saber: La dimensión formal de la política (polity), la dimensión del contenido (policy) y la dimensión del proceso de la política (polics). Ahora bien, se hace necesario establecer una comparación de los modelos estudiados.

\section{COMPARACIÓN DE LOS TRES MODELOS DE COMPETENCIAS Y HALLAZGOS TEÓRICOS}

A través del estudio de la fundamentación de los tres modelos de competencias en formación política desarrollados en Alemania como Austria, se logró identificar una construcción teórica, caracterizado por procesos de profundización, contraste y diferenciación, que, mirando con distancia, nos ofrece una forma de cristalización teórica con relación a qué, por qué y cómo promover procesos formativos en esta temática específica. Con ello me refiero que, si bien los modelos estudiados se han desarrollado cronológicamente en diferentes tiempos, uno en el año 2004, los otros dos en el año 2008 y 2010 respectivamente, y existen entre ellos diferencias cualitativas en sus propuestas, se detecta entre los modelos un hilo conductor que da una cierta direccionalidad a nivel normativo como procedimental.

El nivel normativo está constituido por un conjunto de principios orientadores comunes que a nivel filosófico señalan la intencionalidad y finalidad última que busca este tipo de educación. En cambio, el nivel procedimental hace referencia a la forma o modo en el cual se operacionaliza en términos pedagógicos la concreción de esa meta. Es decir, se refiere a las particularidades del modelo.

En el discurso de la didáctica política, la dimensión normativa está sustentada en la categoría de "polítische Mündigkeit", es decir, "la autonomía política". Los tres modelos estudiados la colocan como la meta más alta de la formación política. Esto significa, por una parte, como condición para el mantenimiento y desarrollo continuo de una cultura política democrática y, por otra, más concreta, como la promoción de capacidades analíticas para elegir desde bases sociales y político-democráticas, durante la socialización escolar. Aquí también es importante subrayar que los tres modelos operacionalizan esta idea de la "politische Mundigkeit" a través de la definición de competencia formulada por F. Weinert, privilegiando, de esta manera, las capacidades analíticas. Por lo tanto, no solo estamos ante la presencia de un concepto filosófico sino también ante una forma de concreción de carácter cognitiva que privilegia capacidades y habilidades cognitivas para resolver problemas. A partir de esta precisión conceptual, se puede comprender el proceso 
de construcción teórica que han generado los modelos estudiados, ese proceso se mueve, por una parte, en dirección al ámbito del saber y, por otra, en dirección a las competencias.

\section{EL ÁMBITO DEL SABER}

Los tres modelos reconocen la importancia del saber dentro del desarrollo de las competencias. Sin embargo, se deja establecido que este saber no tiene un valor en sí mismo, como si se tratase de adquirir conocimientos aislados sobre política, economía o de la sociedad. Por el contrario, en los tres modelos se señala el saber como un "saber de trabajo". En el modelo GPJE se caracteriza como "konzeptuelles Deutungswissen" ("saber de interpretación conceptual"), trata de "un saber que se relaciona con conceptos básicos para la comprensión de la política, la economía y el derecho" (GPJE, 2004, p. 14). Con ello se designa como el estudiante a través del saber estructura sus representaciones y percepciones de política, le permite a través de la interpretación de este saber comprender la lógica interna de las instituciones democráticas y sus problemas. El documento de la GPJE señala claramente que la mediación del saber contribuye al desarrollo de la competencia, pero también el mejoramiento de la competencia contribuye al ampliación y mejoramiento del saber (Cf. GPJE, 2004, p. 14), mostrando una relación interna entre competencia y saber.

Ahora bien, esta primera aproximación de la GPJE no explica el contenido de ese saber. Los otros dos últimos modelos reconocen la función y complejidad que posee el saber dentro el modelo por competencias en la formación política.

Para el modelo de Weißeno, el saber ocupa un rango de dimensión específica de la competencia política, entendida como una dimensión disciplinar que se relaciona con el contenido. El aporte de este trabajo se encuentra en la fundamentación de los conceptos base y conceptos disciplinares. A nivel de conceptos base se puede identificar una distinción entre el modelo de competencia política y el modelo de competencias estructural austriaco de competencias políticas. El primer modelo, planteado por el equipo de Weißeno identifica los conceptos base: "orden”, "decisión" y "bien común”; en cambio, el segundo modelo, sustentado por Krammer (2013) apunta a las tres dimensiones de la política: "polity", "policy", "politics". Ahora bien, surge la pregunta si estas dos propuestas se contraponen o complementan. Klaus Kremb (2010, p. 13) ha mostrado que estos dos modelos de concepto base, en vez de contraponerse, se complementan. Debido que las dimensiones de la política (polity, policy, politics) se ordenan analíticamente en conocimientos teórico didácticos, organizados en orden, decisión y bien común.

La discusión del saber dentro los modelos de competencias en formación política ha permitido visibilizar una forma de concreción de los conceptos base y una forma como se articula la ciencia política y la psicología del aprendizaje mediada por la didáctica política. El modelo Austriaco ofrece una concreción más compleja del saber, presenta, por una parte, la categoría de saber de trabajo y, por otro, propone una competencia denominada "Sachkompetenz", que la traducimos como una "competencia de la estructuración del saber". Con el saber de trabajo, los estudiantes utilizan conceptos, teorías y modelos que medien el saber para el desarrollo de competencia y con la "competencia de la estructuración del saber" 
apunta a la promoción de capacidades, habilidades y disposiciones que son necesarios para comprender términos, categorías o conceptos de la política y sobre ella poder desarrollar una actitud crítica del tema estudiado.

La discusión y contrastación teórica desarrollado en los modelos de competencias germanos nos permite inferir que la dimensión del saber es un componente nuclear y que su comprensión y promoción no se reduce a una cuestión descriptiva de identificar nombres y fechas, sino, por el contrario, promover de manera sistemática las capacidades analíticas y ellas requieren la definición y fundamentación de determinados conceptos base.

\section{DE CAPACIDADES A ÁMBITOS DE COMPETENCIAS}

Un segundo hallazgo del debate de la didáctica política germana está en su opción de profundizar la competencia del juicio político. Su concreción supone el desarrollo de un conjunto de capacidades analíticas. En cambio, las otras competencias como ser la competencia de acción política y competencia metodológica tienen como finalidad contribuir a la promoción del juicio político. Ahora bien, mientras el modelo alemán propuesto por la GPJE publicado el 2004, se refiere en sentido estricto a capacidades (capacidades del juicio político, capacidades de la acción política y capacidades metodológicas). En cambio, el modelo austriaco, publicado el 2008, apunta a competencias (competencia del juicio político, competencia de acción política y competencia metodológica). De ello se desprende que el primer modelo no apunta a varias competencias, sino comprende la existencia de una competencia de formación política, que posee varias dimensiones, las cuales son descritas como capacidades. En cambio, el modelo austriaco nos permite pensar que nos encontramos frente no a una competencia, sino a un ámbito de competencias de la formación ciudadana, por ello se puede hablar de una competencia del juicio político, competencia de la acción política, competencia metodológica y al interior de cada una de ellas se pueden identificar subcompetencias, señalando con ello la complejidad de la formación política.

\section{APORTES DE LA FORMACIÓN POLÍTICA A LA FORMACIÓN INICIAL DE PROFESORES}

Antes de presentar los aportes de la formación política se caracterizan sintéticamente algunos puntos sobre la formación inicial de profesores en la especialidad de formación ciudadana en Chile. Se entiende por formación inicial docente, al igual que todo procedimiento intencional de producción y transmisión de saberes y habilidades, como un proceso de enseñanza-aprendizaje con el que se intenta conseguir algún efecto (finalidades de la formación); en el que se pretende transmitir conceptos, teorías y desarrollar, destrezas o habilidades didácticas (contenidos de la formación); donde los contenidos se tratan de vehicular a través de determinadas acciones (actividades de enseñanza-aprendizaje); y en el que se comprueban los resultados obtenidos con finalidades pedagógicas y/o de certificación (evaluación) (Muñoz, 2011, p. 389). 
Chile diseñó políticas educativas y mecanismos normativos dirigidos a fortalecer la formación inicial docente desde la década de los noventa (Castro Rubilar, 2017; Cisternas, 2011; Ferrada, Villena y Pino, 2018). Con la aprobación de los "Estándares Orientadores para carreras de pedagogía en educación Básica" (Chile, 2011) y "Estándares Orientadores para carreras de pedagogía en educación Media" (Chile,2012) se consignan algunos estándares para promover ciertos conocimientos, habilidades y capacidades relacionados con la formación ciudadana en la formación inicial de profesores.

En la educación básica se establecen dos estándares. El primero apunta a un nivel conceptual: "Comprende los conceptos fundamentales de las ciencias sociales relacionado con formación ciudadana, identidad cultural y organización económica” (Chile, 2011, p. 131). En cambio, el segundo está dirigido a desarrollar capacidades y habilidades: "Es capaz de promover en los estudiantes competencias cívicas y ciudadanas para la democracia” (Chile, 2011, p. 133).

En la educación media se propone un estándar disciplinario, exclusivo para la enseñanza de la Historia, Geografía y Ciencias Sociales: "Desarrolla estrategias didácticas que promuevan los aprendizajes, valores y conductas ciudadanas, conducentes a hacer de sus estudiantes sujetos activos y responsables ante el desafío de la convivencia democrática y desarrollo sustentable" (Chile, 2012, p. 162). A pesar de que han pasado ocho años no hemos encontrado en la literatura consultada estudios sistemáticos que examinen cómo se promueve la formación ciudadana en los centros de formación docente, solo existen algunas aproximaciones (Cárcamo, 2008; Muñoz et al., 2018).

Ahora bien, con la promulgación de Ley 20.911 el año 2016 en Chile, la formación ciudadana ha adquirido relevancia a nivel político y educativo. La Ley establece la creación de un Plan de Formación Ciudadana en los establecimientos escolares. El Ministerio de Educación ha elaborado documentos para orientar la implementación de ese plan en las escuelas (Chile, 2016a, 2016b, 2017). En ese marco, se define la formación ciudadana en los siguientes términos.

Proceso formativo continuo que permite que los niños, niñas, jóvenes y adultos desarrollen un conjunto de conocimientos, habilidades y actitudes que resultan fundamentales para la vida en una sociedad democrática. Busca promover en distintos espacios, entre ellos las comunidades educativas, oportunidades de aprendizaje que permitan que niños, niñas, jóvenes y adultos se formen como personas integrales, con autonomía y pensamiento crítico, principios éticos, interesadas en lo público, capaces de construir una sociedad basada en el respeto, la transparencia, la cooperación y la libertad. Asimismo, que tomen decisiones en consciencia respecto de sus derechos y de sus responsabilidades en tanto ciudadanos y ciudadanas. (Chile, 2016b, p. 11)

A partir de este concepto se extraen cuatro ejes, a saber: "Cultura política democrática"; "pensamiento crítico"; "valores éticos y cívico - democráticos", y "participación activa" que se enmarca dentro la concepción de una ciudadanía activa, crítica y responsable (Chile, 2016a). El concepto de formación ciudadana apunta a 
promover la capacidad para la acción responsable y solidaria dentro la comunidad política democrática, así como el pensamiento crítico dirigido a la emisión de juicios y toma de decisiones. Se reconoce que los estudiantes deben desarrollar no solo conocimientos, sino también actitudes y comportamientos que les permitan resolver problemas en situaciones diferentes dentro la sociedad democrática. Estos componentes son análogos al significado que se encuentra en los conceptos de política de los modelos de competencias de formación política, que apuntan a "promover ciudadanos autónomos, con capacidad de elegir con bases sociales y político democráticas".

Ahora bien, ¿qué aporta la discusión teórica sobre los modelos de formación política a la formación inicial docente para la formación ciudadana? Aquí nos referiremos a dos aportes, que pasamos a describir brevemente.

\section{EL ÁMBITO DEL SABER COMO COMPONENTE NUCLEAR PARA LA FORMACIÓN INICIAL DOCENTE EN FORMACIÓN CIUDADANA}

Uno de los principales aportes que realiza la discusión de la formación política a la formación inicial docente en formación ciudadana es la importancia que le atribuyen al ámbito del saber como condición para promover una competencia.

$\mathrm{El}$ ámbito del saber contiene varios niveles, facetas y funciones que articula, por una parte, el manejo de conceptos, teorías y modelos de las ciencias sociales para explicar determinados fenómenos y, por otro, es atenta a los proceso de aprendizaje, que explican cómo los niños, adolescentes y adultos establecen relaciones conceptuales a partir del cual interpretan esos fenómenos estudiados, sustentado todo ello en un plano normativo, es decir, se sostiene que este saber no se reduce a reproducir conceptos de la política, economía, filosofía, sociología o derecho, sino que tiene un propósito formativo, promover la autonomía política, la responsabilidad, solidaridad y participación como ciudadano dentro de una sociedad democrática. En ese marco, la pregunta que se desprende para la formación inicial docente en formación ciudadana es clarificar ¿Cuál es el saber que debe poseer el profesor para promover la formación ciudadana?, ¿la formación ciudadana posee un estatuto epistemológico?, ¿qué capacidades y habilidades debe desarrollar el profesor de esta asignatura?, ¿qué herramientas metodológicas, didácticas e investigativas debe poseer el educador?, ¿cuál es el rol del profesor en la escuela y la comunidad?

La formación inicial docente en formación ciudadana requiere de competencias relacionadas con el saber de la especialidad, ella hace alusión al manejo de conceptos de la especialidad, así como teorías y modelos de interpretación que le permiten al estudiante comprender y analizar la situación política, económica, social y cultural de la sociedad democrática, tanto a nivel local como global (Detjen, 2007; Sander, 2007). Para ello se requiere el conocimiento interdisciplinar sobre la formación ciudadana, capacitar a los futuros educadores en la promoción de competencias docentes en la especialidad de formación ciudadana.

Para concretar este propósito se requiere a nivel curricular diseñar una asignatura de fundamentos de formación ciudadana, cuya meta del curso es explicar las bases a nivel histórico y teórico de la formación ciudadana como ámbito de 
estudio, ofrecer herramientas teóricas para conceptualizar, caracterizar y diferenciar la formación ciudadana de otras formas de educación política emergentes históricamente en la modernidad, analizar tanto las competencias de la formación ciudadana y sus modelos más representativos como la estructura y mediaciones curriculares, pedagógicas y evaluativas de las políticas educativas en relación a la formación ciudadana en Chile, así como conocer y utilizar las dimensiones de la política y los conceptos base de la especialidad. Este proceso requiere potenciar la capacidad reflexiva del futuro profesor.

\section{ÁMBITO DE COMPETENCIA DIDÁCTICA EN FORMACIÓN CIUDADANA}

Un aspecto relevante que se infiere de los modelos estudiados es que permiten organizar el ámbito disciplinar con el ámbito didáctico, ofreciendo a los profesores herramientas teóricas, metodológicas y didácticas necesarias para promover los aprendizajes en formación ciudadana. Esto significa que la discusión germana sobre los modelos de la formación política pone sobre la mesa la necesidad de fundamentar competencias profesionales de la formación docente en la especialidad de formación ciudadana, ello como condición para promover las competencias ciudadanas en las escuelas.

El modelo de competencias para profesores en formación ciudadana puede hacer más claro la manera de alcanzar esos aprendizajes y conocer las condiciones necesarias para mejorar los procesos de enseñanza y aprendizaje.

Un aspecto importante de los modelos estudiados estriba en promover las capacidades analíticas para comprender y analizar la sociedad democrática. Los modelos estudiados privilegian la competencia del juicio político como la forma para promover esa capacidad crítica.

La UNESCO en su estudio "Educación para la ciudadanía mundial. Temas y objetivos de aprendizaje”, publicado en el 2015 apunta a esta dirección, cuando plantea a lado de la dimensión socioemocional y conductual, la importancia de la promoción de las capacidades analíticas para comprender y analizar los problemas locales, nacionales y mundiales de la sociedad democrática (Cf. UNESCO, 2015, p. 14-16). El presupuesto de esa educación se encuentra en la promoción de la autonomía y participación del ciudadano en la comunidad democrática y no en la consolidación de un Estado nacional como fin en sí mismo. En ese marco, Jürgen Habermas (1999) ha afirmado que la fuente de legitimación de la obligación política es la voluntad de los destinatarios de las leyes. Los destinatarios de derecho deben autocomprenderse como autores del mismo, como ciudadanos libres e iguales que aceptan los principios de la Justicia. Ello nos conduce a promover una formación ciudadanía democrática o formación política con sentido crítico y socialmente conectado, éticamente responsable, respetuoso de la diversidad y comprometido con la sociedad democrática a escala global.

A partir de las competencias de formación política, los requerimientos de la ley 20.911 y documentos orientadores del Plan de Formación Ciudadana se bosqueja en términos generales una propuesta de competencias de formación ciudadana para la formación inicial de profesores. El modelo está compuesto por cinco competencias, ellas son la competencia del juicio político; la competencia comunicativa y 
participativa; la competencia ética ciudadana; la competencia metódica y, finalmente, la competencia relacionada con el saber.

La competencia del juicio político tiene como meta promover la capacidad cognitiva del estudiante a partir de la formulación de un juicio político, su fundamentación, reflexión y evaluación (Detjen, 2007; Sander, 2007). Ello está en relación con el objetivo de "fomentar en el estudiante el ejercicio de una ciudadanía crítica”, así como de promover el conocimiento, comprensión y análisis del Estado de Derecho y de la institucionalidad local, regional y nacional, y la formación de virtudes cívicas en los estudiantes (Chile, 2016a).

La competencia comunicativa y participativa tiene como finalidad promover la capacidad cognitiva y socio-afectiva del estudiante para representar sus propias opiniones, entrar en controversias políticas y poder llegar a compromisos, así como desarrollar formas de comportamiento social que capacitan a las personas para convivir en una sociedad cada vez más plural, relacionándose con los demás, cooperar, comprometerse y afrontar conflitos". Ello se relaciona con el objetivo de "fomentar la participación de los estudiantes en temas de interés público" y "fomentar en los estudiantes la valoración de la diversidad social y cultural” (Chile, 2016a).

La competencia ética ciudadana tiene como meta promover valores éticos y actitudes democráticas en los estudiantes, ello está en relación con la valoración del respeto, el bien común, la tolerancia, la diversidad, la responsabilidad, el pluralismo, la democracia, la transparencia y la probidad, que se relaciona con el objetivo de "Garantizar el desarrollo de una cultura democrática y ética en la escuela", así como "Fomentar una cultura de la transparencia y la probidad" (Chile, 2016a).

Las competencias metódicas, que hacen alusión a las competencias de lectura, planificación y organización de recursos y estrategias didácticas para promover las anteriores competencias (Hellmuth y Klepp, 2010; Sander, 2007).

Las competencias relacionadas con el saber de la especialidad, ella fue descrita en el apartado anterior.

Estos lineamientos del ámbito de la competencia didáctica de la formación ciudadana en la formación inicial de profesores combinan los elementos teóricos, didácticos y organizativos. El futuro profesor requiere poseer conocimientos teóricos sobre la didáctica política como ciencia sinóptica, conocer y saber utilizar los métodos y medios didácticos pertinentes para promover capacidades y habilidades y tener el espacio de desarrollar prácticas en el sistema escolar en el que aplique estas metodologías y reflexione sobre su impacto.

\section{CONCLUSIONES}

Los tres modelos de formación política comparten una misma meta, promover la "politische Mündigkeit", que significa "que cada estudiante sea capaz de desarrollar su propio juicio político, tomar sus propias decisiones y desarrollar su acción responsable dentro la sociedad democrática"(Lobatón, 2016, p. 259). La "politische Mündigkeit" no surge de una disciplina en particular, sino es resultado de una discusión filosófico-política y didáctica de la formación política, comprometida con la formación de un ciudadano activo dentro una sociedad democrática. 
Los tres modelos estudiados operacionalizan la "politische Mündigkeit" como competencia política. Aquí las competencias y los modelos se presentan como mediaciones para alcanzar la meta de formación política. La comprensión de competencias en la línea de Weinert señala claramente que las competencias se entienden como capacidades y habilidades cognitivas, donde la competencia del juicio político adquiere relevancia central dentro las capacidades analíticas. Los componentes de los modelos estudiados priorizan la competencia del juicio político y tanto el ámbito del saber, el ámbito de la competencia de la acción política y el ámbito de la competencia metodológica contribuyen a que los estudiantes puedan analizar y emitir juicios fundamentados con relación a las temáticas estudiadas.

El aporte de la discusión teórica de la formación política a la formación inicial docente en formación ciudadana es de gran relevancia. Ofrece elementos importantes para pensar el saber de la formación ciudadana como un ámbito que posee su propio estatuto epistemológico. Invita a promover procesos formativos en los centros de formación docente que apunten a formar las capacidades analíticas de los ciudadanos, marcando con ello, una distancia cualitativa con la forma de entender las clases de educación ciudadana, centrada aún en un nivel de descripción de las características de las instituciones democráticas.

Un aspecto débil de la propuesta germana es que se concentra solo en el ámbito cognitivo y descuida la dimensión social e intercultural, aspectos necesarios para convivir en las sociedades democráticas actuales.

\section{REFERENCIAS}

CÁRCAMO, H. Importancia atribuída al desarrollo de la ciudadanía en la Formación inicial docente. Estudios Pedagógicos, Valdivia, v. 34, n. 2, p. 29-43, 2008. Disponible en: https://scielo.conicyt.c1/pdf/estped/v34n2/art02.pdf. Acesso en el: 4 jun. 2019.

CASTRO RUBILAR, J. I. La formación inicial docente en Chile: Una parte de su historia y los desafíos de aprendizaje por competencias. Praxis Educativa, Santa Rosa, v. 21, n. 2, p. 12-21, mayo-ago. 2017. https://dx.doi.org/10.19137/ praxiseducativa-2017-210202

CISTERNAS, T. La investigación sobre formación docente en Chile. Territorios explorados e inexplorados. Calidad en la Educación, Santiago, n. 35, p. 131-164, dic. 2011. http://dx.doi.org/10.4067/S0718-45652011000200005

CHILE. Ministerio de Educación (MINEDUC). Estándares Orientadores para carreras de pedagogía en educación Básica. Estándares pedagógicos y disciplinarios. Santiago: MINEDUC, 2011.

CHILE. Ministerio de Educación (MINEDUC). Estándares Orientadores para carreras de pedagogía en educación Media. Estándares pedagógicos y disciplinarios. Santiago: MINEDUC, 2012.

CHILE. Ministerio de Educación (MINEDUC). Orientaciones curriculares para el desarrollo del Plan de Formación Ciudadana. Santiago: MINEDUC, 2016a. 
CHILE. Ministerio de Educación (MINEDUC). Orientaciones para la elaboración del Plan de Formación Ciudadana. Santiago: MINEDUC, $2016 \mathrm{~b}$.

CHILE. Ministerio de Educación (MINEDUC). Orientaciones para la participación en comunidades educativas en el marco del Plan de Formación Ciudadana. Santiago: MINEDUC, 2017.

DETJEN, J. (ed.). Politische Bildung: Geschichte und Gegenwart in Deutschland. München/Wien: R. Oldenburg Verlag, 2007.

DETJEN, J. Basis- und Fachkonzepte der politischen Bildung. Ein Kommentar zu Wolfgang Sanders Thesen. In: GOLL, T. (ed.). Politikdidaktische Basis: und Fachkonzepte. Schwalbach: Wochenschau Verlag, 2011. p. 44-48.

FERRADA, D.; VILLENA, A.; PINO, M.D. ¿Hay que formar a los docentes en políticas educativas? Cadernos de Pesquisa, São Paulo, v. 48, n. 167, p. 254-279, 2018. https://doi.org/10.1590/198053144740

GESELLSCHAFT FÜR POLITIKDIDAKTIK UND POLITISCHE JUGENDUND ERWACHSENENBILDUNG (GPJE). Anforderungen an Nationale Bildungsstandards für den Fachunterricht in der Politischen Bildung an Schulen. Ein Entwurf. 2. Aufl. Schwalbach/Ts: Wochenschau Verlag, 2004.

GOLL, T. (ed.). Politikdidaktische Basis: und Fachkonzepte. Schwalbach: Wochenschau Verlag, 2011.

HABERMAS, J. La inclusión del otro: Estudios de teoría política. Barcelona: Paidós, 1999.

HEDINGER, F. Erkentnisse aus der empirischen Politikwissenschaft für das Politikkompetenzmodell. In: ZIEGLER, B.; WALDIS, M. (eds.). Politische Bildung in der Demokratie. Interdisziplinäre Perspektiven. Wiesbaden: Springer, 2018. p. 57-73.

HELLMUTH,T.; KLEPP, C.Politische Bildung. Geschichte. Modelle. Praxisbeispiele. Wien/Köln: Böhlau Verlag, 2010.

KLAFKI, W. Neue Studien zur Bildungstheorie und Didaktik. Zeitgemäße Allgemeinbildung und kritisch-konstruktive Didaktik. 2.ed.Weinheim/Basel: Beltz, 1991. KRAMMER, R. Kompetenzen durch politische Bildung. Ein KompetenzStrukturmodell. In: WIRTITSCH, M. (ed.). Kompetenz-orientierung. Eine Herausforedrung für die Lehrerbildung. Schwalbach: Wochenschau Verlag, 2013. p. $15-21$.

KRAMMER, R.; KÜHBERGER, C.; WINDISCHBAUER, E. (eds.). Die durch politische Bildung zu erwerbenden Kompetenzen. Ein Kompetenz-Strukturmodell: Ein Projeckt im Rahmen der Demokratie-Initiative der Bundesregierung. Wien: Bundesministerium für Unterricht, Kunst und Kultur, 2008.

KREMB, K. Kompaktwissen Politikdidaktik. Kategorien. Konzeptionen. Kompetenzen. Schwalbach T/s: Wochenschau Verlag, 2010.

KÜHBERGER, C.Zur Genese und Konzeption des österreichischen Kompetenzmodells für die politische Bildung. In: WOCHENSCHAU, S. (ed.). I + II: Kompetenzen im Politikunterricht. Politik und Wirtschaft unterrichten Wochenschau. Schwwalbach: Wochenschau, 2010. p. 44-56. 
LOBATÓN, R. El ámbito científico de la formación política: elementos conceptuales y líneas de investigación. Educación y Educadores, Universidad de la Sabana, v. 19, n. 2, p. 254-273, 2016. http://dx.doi.org/10.5294/edu.2016.19.2.5

MASSING, P. Die Infrastruktur der politischen Bildung in der Bundesrepublik Deutschland - Fächer,Institutionen, Verbände, Träger.In: SANDER, W.(ed.).Handbuch politische Bildung. Bonn: Bundeszentrale für politische Bildung, 2007. p. 62-78.

MASSING, P. Basiskonzepte für die politische Bildung. In: WEIßENO, G. (ed.). Politikkompetenz. Was Unterricht zu leisten hat: Bundeszentrale für politiche Bildung. Bonn, 2008, p. 185-198.

MASSING, P. Basis- und Fachkonzepte zwischen "Fachlichkeit und Interdisziplinarität". In: GOLL, T. (ed.). Politikdidaktische Basis - und Fachkonzepte. Schwalbach: Wochenschau Verlag, 2011. p. 56-70.

MUÑOZ, C. et al.Percepción de los estudiantes que ingresan a estudiar pedagogía de lo que es un buen ciudadano, las instituciones públicas y la efectividad de la acción política. Educación, Lima, v. 27, n. 52, p. 63-80, marzo 2018. http://dx.doi.org/10.18800/ educacion.201801.004

MUÑOZ, F. I. La formación pedagógica del docente universitario. Educação, Santa María, v. 36, n. 3, p. 387-396, sep.-dic. 2011. https://doi.org/10.5902/198464442970

ORGANIZAÇÃO DAS NAÇÕES UNIDAS PARA A EDUCAÇÃO, A CIÊNCIA E A CULTURA (UNESCO). Educación para la ciudadanía mundial. Temas y objetivos de aprendizaje. París: UNESCO, 2015.

ORGANIZAÇÃO DAS NAÇÕES UNIDAS PARA A EDUCAÇÃO, A CIÊNCIA E A CULTURA (UNESCO). La Formación Inicial Docente en Educación para la Ciudadanía en América Latina. Análisis comparado de seis casos nacionales. Estrategia Regional sobre Docentes. Santiago: OREALC-UNESCO, 2017.

REDWANZ, W. Deutschland, Österreich und Schweitz: Drei Kompetenzmodelle zur politischen Bildung auf dem Prüfstand. In: WIRTITSCH, M. (ed.). Kompetenzorientierung. Eine Herausforedrung für die Lehrerbildung. Schwalbach: Wochenschau Verlag, 2013. p. 126-156.

RICHTER, D. Basis- und Fachkonzepte der Politik. Ein konsensuell erarbeitetes Kompetenzmodell. In: WOCHENSCHAU, S. (ed.). I + II: Kompetenzen im Politikunterricht. Politik und Wirtschaft unterrichten Wochenschau, 2010. p. 58-69.

SANDER, W.Politik entdecken-Freiheit leben. Schwalbach:Wochenschau Verlag, 2007. SANDER, W. Was heißt "Rainaissence der Bildung”? Ein Komentar. Zeitschrift für Pädagogik, Weinheim, v. 61, n. 4, p. 517-526, jul./ago. 2015. Disponible en: https:// www.pedocs.de/volltexte/2018/15375/pdf/ZfPaed_2015_4_Sander_Was_heisst_ Renaissance.pdf. Accesso en el: 16 abr. 2019.

WEIßENO, G. Politikkompetenz. Neue Aufgaben für Theorie und Praxis. In: WEIßENO, G. (ed.). Politikkompetenz. Was Unterricht zu leisten hat. Bonn: Bundeszentrale für politiche Bildung, 2008. p. 11-20. 
WEIßENO, G. Dimenzionen der Polikkompetenz.In:WEIßENO, G.; BUCHSTEIN, H. (ed.). Politische Handeln: Modelle, Möglichkeiten, Kompetenen. Bonn: Bundeszentrale für politiche Bildung, 2012, p. 156-177.

WEIßENO, G.; DETJEN, J.; JUCHLER, I.; MASSING, P.; RICHTER, D. Konzepte der Politik - ein Kompetenzmodell. Bonn: Bundeszentrale für politiche Bildung, 2010.

WIRTITSCH,M.Zur Entstehung eines österreichischen Kompetenz-Strukturmodells Politische Bildung. In: WIRTITSCH, M. (ed.). Kompetenz-orientierung. Eine Herausforedrung für die Lehrerbildung. Schwalbach: Wochenschau Verlag, 2013. p. 5-14.

ZIEGLER, B. Legitimationsfiguren der Politischen Bildung. In: ZIEGLER, B.; WALDIS, M. (eds.). Politische Bildung in der Demokratie. Interdisziplinäre Perspektiven. Wiesbaden: Springer, 2018. p. 97-109.

\section{SOBRE EL AUTOR}

Ramiro Gastón Lobatón Patiño es doctor en ciencias de la educación por la Eberhard Karls Universität Tübingen (Alemania). Profesor de la Universidad Católica del Maule (Chile).

E-mail: ramirogastonlobaton@gmail.com

Recibido el 14 de octubre de 2019 Aprobado el 8 de mayo de 2020 